\title{
Design of Electromagnetic and Centrifugal Force Pitch Angle Stall Controller on Application to Small-Scaled Wind Turbine System
}

\author{
Faramarz Asharif ${ }^{1}$, Shiro Tamaki ${ }^{2}$, Teppei Hirata ${ }^{3}$, Tsutomu Nagado ${ }^{4}$ and Tomokazu Nagata ${ }^{5}$ \\ University of the Ryukyus, Faculty of Engineering, \\ Graduate School of Science and Engineering \\ 1-Senbaru, Nishihara, Okinawa, Japan, 903-0213 \\ ${ }^{1}$ faramarz@neo.ie.u-ryukyu.ac.jp \\ ${ }^{2}$ shiro@ie.u-ryukyu.ac.jp \\ ${ }^{3}$ teppei@neo.ie.u-ryukyu.ac.jp \\ ${ }^{4}$ nagado@eee.u-ryukyu.ac.jp \\ ${ }^{5}$ nagayan@ie.u-ryukyu.ac.jp
}

\begin{abstract}
The aim of this research is to evaluate the behavior of small scaled wind turbine system against strong wind input using electromagnetic stall control system. In general, the wind turbine system is generating energy from the revolution of blades. The revolution of blades is depended on the velocity of wind. Therefore, if there is strong wind then the revolution of blade is increased and consequently more energy will be generated. Therefore, generally the wind turbine systems are located in the gale area in order to generate energy efficiency. However, there are some limitations of the revolution of blades or the angular velocity of blades, especially in small-scaled turbine. If the angular velocity exceeds the limit of revolution, then wind turbine system may breakdown. Thus in this paper, in order to avoid the malfunction of small-scaled wind turbine system, electromagnetic stall control (ESC) is suggested. ESC can control the angular velocity without having any connection with shaft. Thus, we would control angular velocity much efficiently than the conventional stall control method such friction stall control. As a consequence, we could verify the reliability of ESC system.
\end{abstract}

Keywords: Wind turbine system, nonlinear control, Pitch angle and Electromagnetic stall control

\section{Introduction}

The purpose of this research is to control the angular velocity of blades in storms condition. In general, the wind turbine system is located in gale area in order to generate energy efficiently. However, in the case of storms, the wind turbine must stop the operation. Thus, energy could not be generated. Hence, in order to avoid this wasteful period, we have suggested the electromagnetic stall control of smallscaled wind turbine which is consist of pitch angle and magnetic field control. By utilization of magnetic and pitch angle control of blade, the angular velocity of blade is controllable even in storm. So, in this paper two different schemes of restrain control [1-4] are proposed. The aim of using magnetic stall control is to decrease the angular velocity by unconnected magnetic friction between blades and turbines shaft. Moreover, pitch angle control is combined with ESC to maintain suitable performances. Next section introduces the conventional pitch angle control and later on ESC system.

\section{Introduction to Small-Scaled Wind Turbine System}

From old times, the utilization of small-sized wind turbine is widely used for pumping up and so on. In the spite of this, in these days the importation of electronic power generator of small scaled wind turbine is not volunteered. The important problem of small scaled wind turbine is the establishment of stall control against strong wind. As a result, there are many excessive rotations that may cause the damage accident. If this problem is solvable, then hereafter it will contribute large amount of energy. Thus, the innovation of small-sized wind turbine is very important part of the energy generation. In this research, the wind turbine system is considered to be located in gale area and control the angular velocity of blade by axis friction and pitch angle. The wind turbine rotates clockwise from the front perspective. In general, the blades of wind turbine descend 3-5 degree to the front and lay on the center axis with a slant of 2-4 degree. The axis is propped up by 2 springs which cross to the right angle of center axis. In this moment, pitch angle is optimized. The stall control mechanism is as follows. At first, in windless or calm, the attitude of blade is on axis. When wind turbine receive the strong wind to the area of swept by blade, the blades are strongly propelled to the backward. Subsequently, the blades reach to stall angle and angular velocity is reduced. Secondly, when turbine receive strong wind, the centrifugal force will increase and with that force, the axis of blades shifted to the central axis line and simultaneously the slop angle becomes reverse of initial value of pitch angle that reduces the angular velocity. These stall control dynamics are possible to realize by the movement of the blade to backward or forward and independently axis rotation. Eventually, the stall control is realized and angular velocity of blade is reduced and by this procedure, centrifugal force is vanished and the mechanism of blades, return to its origin position and it will start to rotate again. In other words, by passive control of ESC to the blades, mechanism of stall control is realizable. Basically, ESC is adjustable by the generated current. The mechanism of ESC is that when angular velocity exceeds the boundary then current is fed back to turbines shaft then at that point it propagates the 
electromagnetic field so the rotating shaft will be stalled by the magnetic field. This procedure will circulate recursively until the angular velocity reach to under the boundary revolution. Thus the combination of pitch angle and electromagnetic stall control can be maintained the stability and good performances even in the storm. In the next chapter the configuration of small-scaled wind turbine system is mentioned.

\section{Modeling of Small-Scaled Wind Turbine System}

As we have introduced small-scaled wind turbine system has many advantages [1-3] since it is small and has low cost for maintenance. Thus, let us be more familiar with its dynamics. In the following, dynamics of horizontal-axis of small-scaled wind turbine system with pitch angle and passive friction control are indicated.

\section{Dynamics of angular velocity}

$\left(J_{\omega}+I_{G}\right) \frac{d \omega(t)}{d t}=\frac{1}{2} C_{T} \rho A R \int_{0}^{t} V^{2}(t) e^{-(\tau-t)} d \tau \Theta(\theta)-\frac{1}{2} I_{G} \omega^{2}(t)$

$-f_{r} \omega(t)$

Dynamics of pitch angle

$J_{b} \frac{d^{2} \theta(t)}{d t^{2}}=M R^{2} \omega^{2}(t) \theta(t)-L \theta(t) k-c \frac{d \theta(t)}{d t}$

which we have:

$\Theta(\theta)=\frac{\widetilde{\theta}(t)}{\theta_{\max }}$ and $\tilde{\theta}(t)=\theta_{\max }-\theta(t)$.

Where, the parameters are indicates in the below table 1 .

Table 1. Identification of parameters

\begin{tabular}{|c|c|}
\hline$J_{\omega}$ & Inertia moment of blade \\
\hline$\omega(t)$ & Angular velocity \\
\hline $\mathrm{C}_{\mathrm{T}}$ & Torque factor \\
\hline$\rho$ & Density of atmosphere \\
\hline $\mathrm{A}$ & Area swept by blade \\
\hline $\mathrm{R}$ & Length of blade \\
\hline $\mathrm{V}(\mathrm{t})$ & Speed of wind \\
\hline$\Theta(\theta)$ & $\begin{array}{c}\text { Power Generation Inertia } \\
\text { Moment Factor }\end{array}$ \\
\hline $\mathrm{I}_{\mathrm{G}}(\mathrm{T})$ The axis friction factor \\
\hline $\mathrm{f}_{\mathrm{r}}$ & Inertia moment of a plate of blade \\
\hline$J_{b}$ & Mass of a blade \\
\hline $\mathrm{M}$ & $\begin{array}{c}\text { The distance from spring to the } \\
\text { edge of blade }\end{array}$ \\
\hline $\mathrm{L}$ & Factor of spring \\
\hline $\mathrm{k}$ & damper \\
\hline $\mathrm{c}$ & Initial values of pitch angle \\
\hline$\theta_{\max }$
\end{tabular}

As it is clear in equation (1), the input wind includes with input delay and convolute with a first order filter. The convolution is done due to blade's moment inertia of turbine. The next term to the convolution is generator torque's lost. Since generator is loaded on the shaft, it would become a load against the blade's rotation. Thus subtraction is done. The next term to the torque's load is axis friction between the shaft and blades conjunction. Since we have mechanical connection between shaft and blade's axis we have to consider the energy lost at this point as well. In equation 2 pitch angle dynamics consist of correlation of pitch angle and energy of angular movement subtracted by shock absorber loads. Through equation (2) the wind turbine stall factor $\Theta(\theta)$ is fed backed to equation (1) and by this procedure the angular velocity is controlled. Following shows the illustration of small-scaled wind turbine and the actual one.

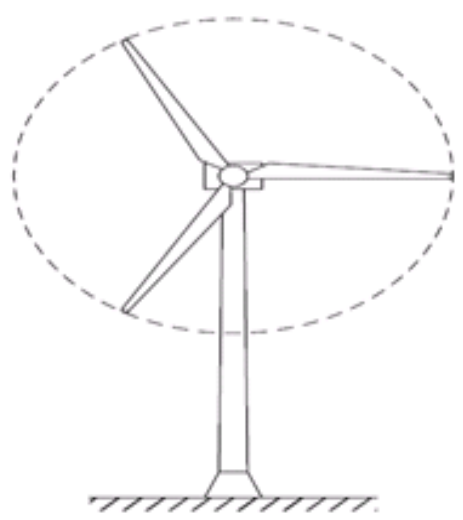

Fig. 1. Illustration of small-scaled wind turbine

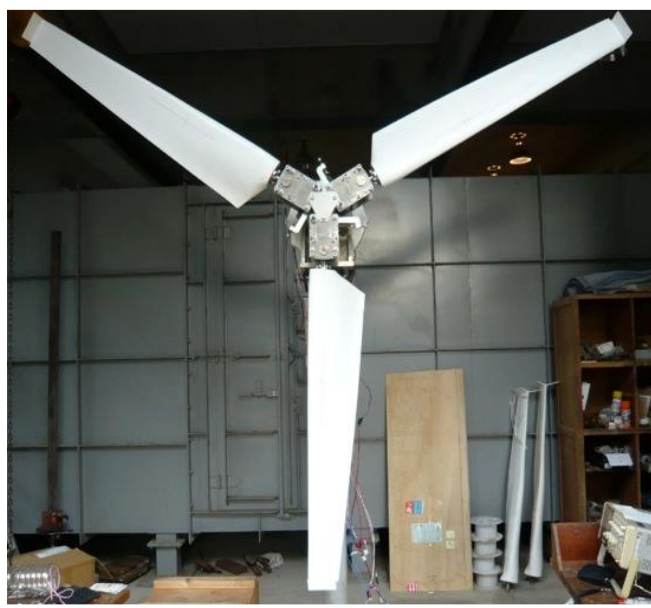

Fig. 2. The actual small-scaled wind turbine

As it is obvious in Fig. 2, small-scaled wind turbine system can be located and installed in general residential property and since it has short blade compare to conventional wind turbine, angular velocity can be controlled by pitch angle and axis friction. 


\section{Adaptive Control of Axis Friction}

As we have discussed in the previous chapter angular velocity can be controlled by combination of friction and pitch angle control. However, unlike the pitch angle control friction control is not adaptable in this status. Therefore, in order to operate small-scaled wind turbine even in extremely strong wind situation, friction loads should be feasible. Otherwise, operation cannot be allowed since if angular velocity exceeds the boundary velocity then it may cause the malfunction of entire system. Thus, to avoid any instability and malfunction, adaptive friction control is proposed. By adaptive friction control, it can adaptively adjust the coefficient of friction value. On the other hand adaptive friction control could be more efficient than passive since when the wind is not strong then friction can be released so generator can produces energy more efficiently. Following shows the mathematical expression of implementation of adaptive friction control in dynamics of blade's angular velocity.

By rewriting the equation (1) the dynamics of small-scaled wind turbine system of angular velocity can be indicated as follows that if angular velocity exceed the boundary, the adaptive friction $\gamma(\omega(t))$ loaded and decrease the angular velocity. Otherwise $\gamma(\omega(t))$ has no influence.

$$
\gamma(\omega(t))=1+e^{\omega(t)-\omega_{\lim i t}}
$$

\section{Dynamics of angular velocity with adaptive}

\section{friction}

$\left(J_{\omega}+I_{G}\right) \frac{d \omega(t)}{d t}=\frac{1}{2} C_{T} \rho A R \int_{0}^{t} V^{2}(t) e^{-(\tau-t)} d \tau \Theta(\theta)-\frac{1}{2} I_{G} \omega^{2}(t)$ So, by choosing the $\alpha\left(\omega^{2}(t)\right)=\frac{J_{b}+M R \omega^{2}(t)}{L k}$,

$-f_{r} \gamma(\omega(t)) \omega(t)$

Also in order to have better performances, pitch angle control is required. Therefore, first we linearize the equation (2). Here, we fix the angular velocity. Then we have:

\section{Dynamics of pitch angle}

$$
J_{b} \frac{d^{2} \theta(t)}{d t^{2}}=M R^{2} \omega^{2} \theta(t)-\alpha L \theta(t) k-c \frac{d \theta(t)}{d t}
$$

\section{Laplace transform}

$J_{b}\left(s^{2} \theta(s)-s \theta(0)-\dot{\theta}(0)\right)=$

$\left(M R^{2} \omega^{2}-\alpha L k\right) \theta(s)+c(s \theta(s)-\theta(0))$

$G_{\theta}(s)=\frac{(1-s) \theta(0)}{s^{2}+\frac{c}{J_{b}} s+\frac{L k \alpha-M R \omega^{2}}{J_{b}}}$

$\dot{\theta}(0)=0$ and $\theta(0)=\theta_{\text {max }}$.

Here, if $\alpha=\frac{J_{b}+M R \omega^{2}}{L k}$ and substitute to equation (6),

then we have: $G_{\theta}=\frac{(1-s) \theta(0)}{s^{2}+\frac{c}{J_{b}} s+1}$.

According to final value theorem the steady step response is:

$\theta_{\text {steady }}=\theta(\infty)=\lim _{s \rightarrow 0} s G_{\theta} \frac{1}{s}=\theta(0)$ the performance of turbine is improved.

As it is clear equation (2) reformed to equation (4) for adaptive friction control and pitch angle control factor is obtained by linearizing the equation (2) and eventually using the final value theorem we have chosen the factor $\alpha$ as it is shown on above. By these two proposed method, it can expect the performance improvement maintaining the stability of entire system. Following shows the block diagram of proposed method.

The Laplace transform of above equation is: 


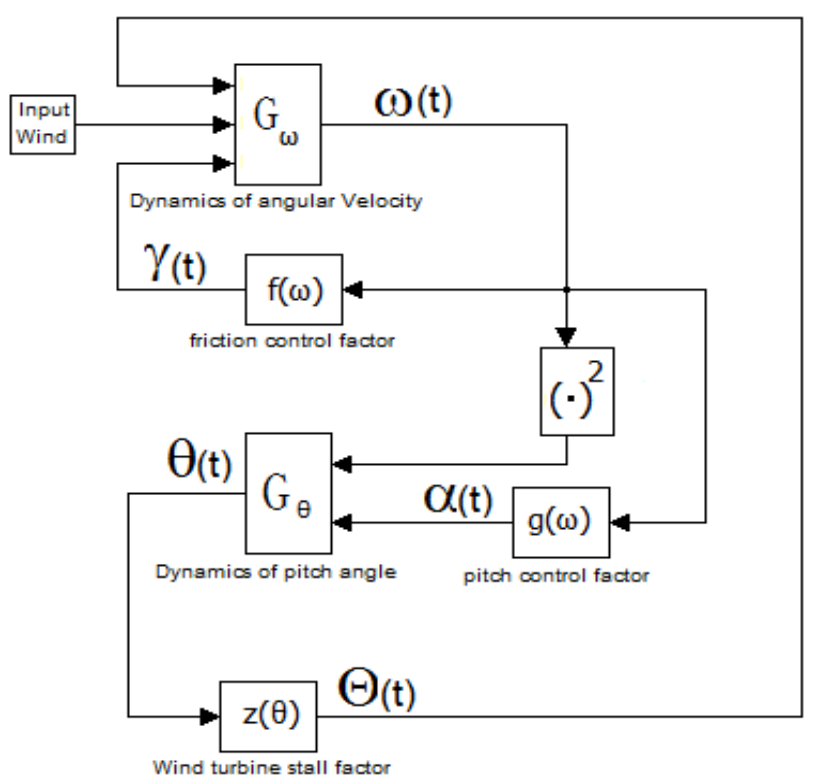

Fig.3. Block diagram of adaptive friction control and pitch angle control

Operation factor in fig. 3 is indicates as below:

$$
\begin{gathered}
f(\omega)=1+e^{\left.\omega(t)-\omega_{\mathrm{lim}}\right)} \\
z(\theta)=\frac{\theta_{\max }-\theta(t)}{\theta_{\max }} \\
g(\omega)=\frac{J_{b}+M R \omega^{2}}{L k}
\end{gathered}
$$

Above block diagram shows the adaptive friction control and performance control of wind turbine. As it is shown, the friction control factor and pitch control factor are controlled by observing the angular velocity of blade. Finally the wind turbine stall factor is fed back to dynamics of angular velocity of blade. By this circulation angular velocity is controlled. In results we will see the behavior of angular velocity and pitch angle of blade for passive control and adaptive control. Here, the reference angular velocity is set to $30[\mathrm{rad} / \mathrm{s}]$. The input wind is considered to be a Rayleigh distribution since variance of wind can be varied with respect to environment and time. The average speed of wind considered to be $50[\mathrm{~m} / \mathrm{s}]$ that in ordinary situation small-scaled wind turbine must stop running. The simulation process is to observe the angular velocity of blade and pitch angle of blade for passive and adaptive control of friction in two cases, one for pitch angle with damper and without damper. Finally compare the results of adaptive and passive control and analysis the behavior and performance of wind turbine system.

\section{Introduction to Electromagnetic Stall Control System}

In this chapter we are going to introduce electromagnetic stall control method. Electromagnetic stall control method [4$5]$ is aimed to control the angular velocity by force which occurred by electromagnetic field and using electromagnetic field shaft's rotation can be suppressed. This matter can be done without having any mechanical connection. Thus, the advantages of electromagnetic stall control are first since there is no mechanical connection or collision between shaft and friction energy lost is minimized and secondly maintenance is also simplified. Now, let us see how does electromagnetic stall controls work. Following shows the mathematical expression of electromagnetic stall control. Dynamics of horizontal-axis wind turbine with pitch angle control is indicated as follows.

$$
\begin{aligned}
& \left(J_{\omega}+I_{G}\right) \frac{d \omega(t)}{d t}=\frac{1}{2} C_{T} \rho A R \int_{0}^{t} V^{2}(t) e^{-(\tau-t)} d \tau \Theta(\theta) \\
& -\frac{1}{2} I_{G} \omega^{2}(t)-f_{B}(t) S(\omega(t)) \\
& B(t)=\mu H(t) \\
& \downarrow \\
& H(t)=\frac{i(t)}{2 r} N \\
& \text { Then the force becomes as follows }
\end{aligned}
$$

$f_{B}(t)=B(t) i(t) l=\mu \frac{N l}{2 r} i^{2}(t)$.

Subsequenty, we would have:

$$
\begin{aligned}
& \left(J_{\omega}+I_{G}\right) \frac{d \omega(t)}{d t}=\frac{1}{2} C_{T} \rho A R \int_{0}^{t} V^{2}(t) e^{-(\tau-t)} d \tau \Theta(\theta) \\
& -\frac{1}{2} I_{G} \omega^{2}(t)-\mu \frac{2 r l}{N} i^{2}(t) S(\omega(t)) .
\end{aligned}
$$

The decision function can be defiend as follows

$S(\omega(t))=\frac{1}{\pi} \tan ^{-1}\left[\alpha_{\text {adj }}\left(\omega(t)-\omega_{\text {stall }}\right)\right]+\frac{1}{2}$.

Then from the turbine generator we can retrive the following current $\rightarrow \frac{1}{2} I_{G} \omega^{2}(t)=K i(t)$. 
Eventually, we would have following equations for pitch angle control and Electromagnetic stall control system.

$$
\begin{aligned}
& \left(J_{\omega}+I_{G}\right) \frac{d \omega(t)}{d t}=\frac{1}{2} C_{T} \int_{0}^{t} \int_{0}^{t} V^{2}(t) e^{-(\tau-t)} d \tau \Theta(\theta) \\
& -\frac{1}{2} I_{G} \omega^{2}(t)-\mu \frac{N I_{G}^{2} l}{2 r K^{2}} \omega^{4}(t) S(\omega(t)) \\
& J_{b} \frac{d^{2} \theta(t)}{d t^{2}}=M R^{2} \omega^{2} \theta(t)-\alpha L \theta(t) k-c \frac{d \theta(t)}{d t}
\end{aligned}
$$

which we have:

$$
\Theta(\theta)=\frac{\widetilde{\theta}(t)}{\theta_{\max }} \text { and } \tilde{\theta}(t)=\theta_{\max }-\theta(t) \text {. }
$$

\begin{tabular}{|c|c|}
\hline$J_{\omega}$ & Inertia moment of blade \\
\hline$\omega(t)$ & Angular velocity \\
\hline $\mathrm{C}_{\mathrm{T}}$ & Torque factor \\
\hline$\rho$ & Density of atmosphere \\
\hline $\mathrm{A}$ & Area swept by blade \\
\hline $\mathrm{R}$ & Length of blade \\
\hline $\mathrm{V}(\mathrm{t})$ & Speed of wind \\
\hline$\Theta(\theta)$ & Wind turbine stall factor \\
\hline $\mathrm{I}_{\mathrm{G}}$ & $\begin{array}{c}\text { Power Generation Inertia Moment } \\
\text { Factor }\end{array}$ \\
\hline$f_{r}$ & The axis friction factor \\
\hline$J_{b}$ & Inertia moment of a plate of blade \\
\hline M & Mass of a blade \\
\hline$f_{B}$ & Electromagnetic stall factor \\
\hline $\mathrm{k}$ & Factor of spring \\
\hline $\mathrm{c}$ & damper \\
\hline $\mathrm{S}$ & Decision function \\
\hline $\mathrm{B}$ & Magnetic field \\
\hline $\mathrm{H}$ & Auxiliary magnetic field \\
\hline$i$ & Generator Current \\
\hline$K$ & Torque constant \\
\hline$\mu$ & Permeability of air \\
\hline $\mathrm{N}$ & Number of wire turns \\
\hline 1 & Length of the coil \\
\hline $\mathrm{r}$ & Thickness of coil \\
\hline$\omega_{\text {stall }}$ & Boundary of angular velocity \\
\hline$\alpha_{\text {adj }}$ & Decision Functional adjustment \\
\hline
\end{tabular}

Where, the parameters are indicated in the below Table 2 .

Table 2. Identification of parameters

In the following Fig.4, it shows the ECS configuration. As it is clear, $\omega(\mathrm{t})$ can be measured and if $\omega(\mathrm{t})$ exceeds the boundary then by decision function current will be flow to the actuator and as result we would have reduced $\omega(\mathrm{t})$ which is $\omega(\mathrm{t})^{\prime}$.

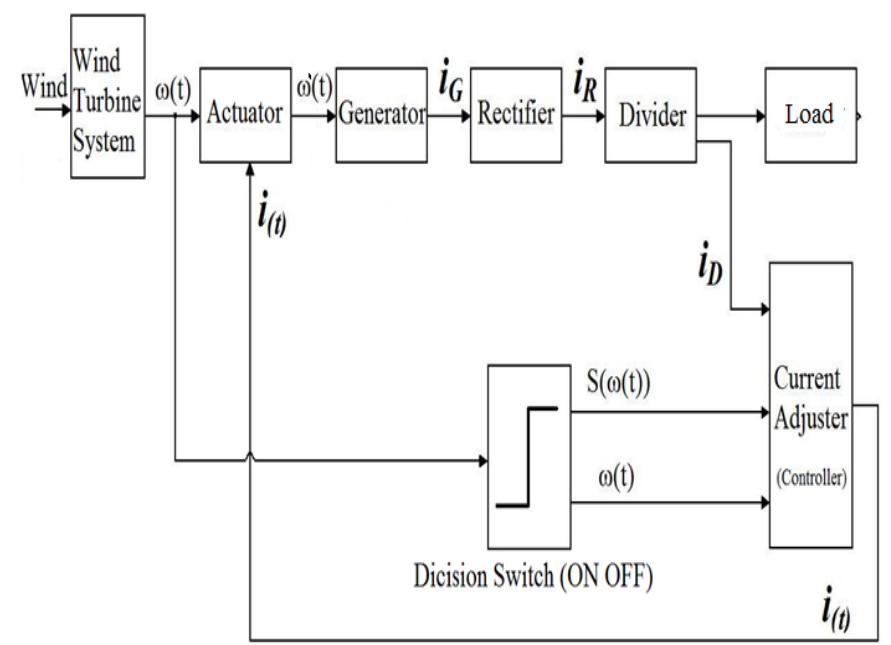

Fig.4. ECS configuration

\section{Simulation and Results}

In order to verify the feasibility of ESC system we have simulated and compared with conventional adaptive control.

\begin{tabular}{|c|c|c|}
\hline Parameters & Values & Units \\
\hline$J_{\omega}$ & 16 & $\mathrm{~kg} \cdot \mathrm{m}^{2}$ \\
\hline$\omega(t)$ & Variable & $\mathrm{rad} / \mathrm{s}$ \\
\hline $\mathrm{C}_{\mathrm{T}}$ & 0.2 & $\mathrm{~N} \cdot \mathrm{m}^{2}$ \\
\hline$\rho$ & 1.2 & $\mathrm{~kg} / \mathrm{m}^{3}$ \\
\hline $\mathrm{A}$ & 7.0686 & $\mathrm{~m}^{2}$ \\
\hline $\mathrm{R}$ & 1.5 & $\mathrm{~m}$ \\
\hline $\mathrm{V}(\mathrm{t})$ & Reyleigh distribution & $\mathrm{m} / \mathrm{s}$ \\
\hline$\Theta(\theta)$ & Variable & - \\
\hline $\mathrm{I}_{\mathrm{G}}$ & 4.5185 & $\mathrm{~A}$ \\
\hline$f_{r}$ & 0.001 & $\mathrm{~kg} \cdot \mathrm{m}^{2} / \mathrm{s}$ \\
\hline$J_{b}$ & 8 & $\mathrm{~kg} \cdot \mathrm{m}^{2}$ \\
\hline $\mathrm{M}$ & 14 & $\mathrm{~kg}$ \\
\hline$f_{B}$ & Variable & $\mathrm{N} \cdot \mathrm{m}$ \\
\hline $\mathrm{k}$ & 100 & $\mathrm{~N} / \mathrm{m}^{2}$ \\
\hline $\mathrm{c}$ & 10 & $\mathrm{~kg} \cdot \mathrm{m}^{2} / \mathrm{s}$ \\
\hline $\mathrm{S}$ & 0 or 1 & - \\
\hline $\mathrm{B}$ & Variable & $\mathrm{Wb} / \mathrm{m}^{2}$ \\
\hline $\mathrm{H}$ & Variable & $\mathrm{A} / \mathrm{m}$ \\
\hline$i$ & Variable & $\mathrm{A}$ \\
\hline$K$ & 0.1 & $\mathrm{~N} \cdot \mathrm{m}$ \\
\hline$\mu$ & $4 \pi \times 10^{-7}$ & $\mathrm{~N} / \mathrm{A}^{2}$ \\
\hline $\mathrm{N}$ & 100 & - \\
\hline 1 & 1 & $\mathrm{~m}$ \\
\hline $\mathrm{r}$ & 0.1 & $\mathrm{~m}$ \\
\hline$\omega_{\text {stall }}$ & 25 & $\mathrm{rad} / \mathrm{s}$ \\
\hline$\alpha_{\text {adj }}$ & 5 & - \\
\hline
\end{tabular}
Let us determine the parameters of system. Following table shows the parameters value.

Table 3. ESC parameters 


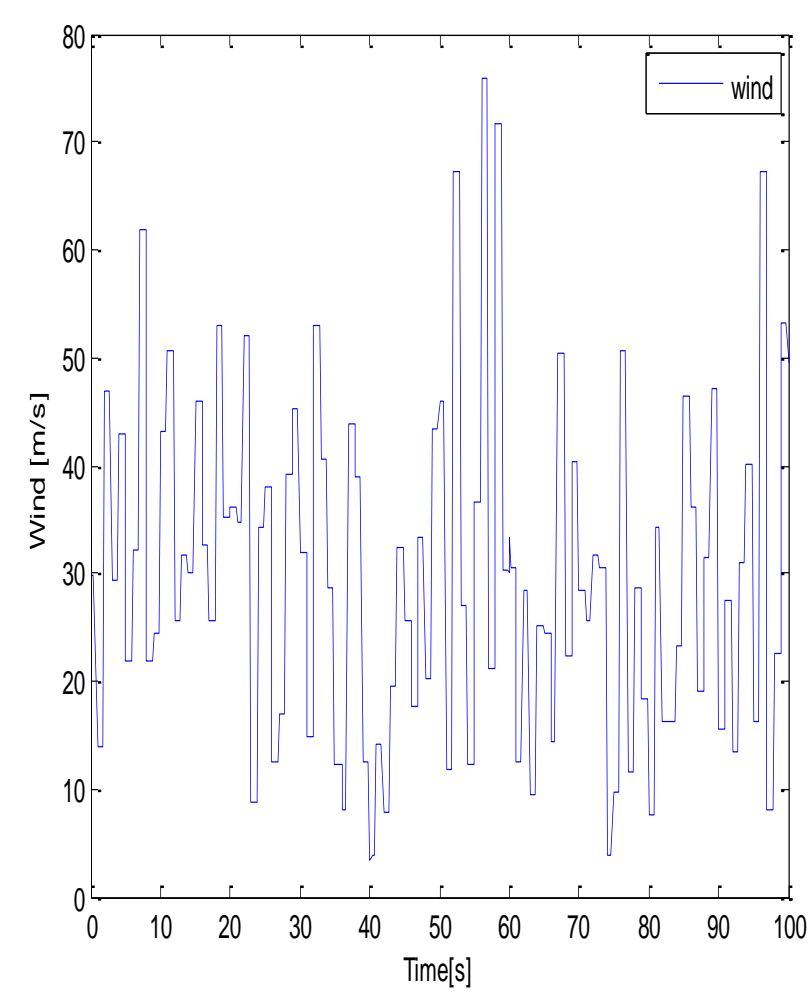

Fig.6. ECS Input wind to small-scaled wind turbine

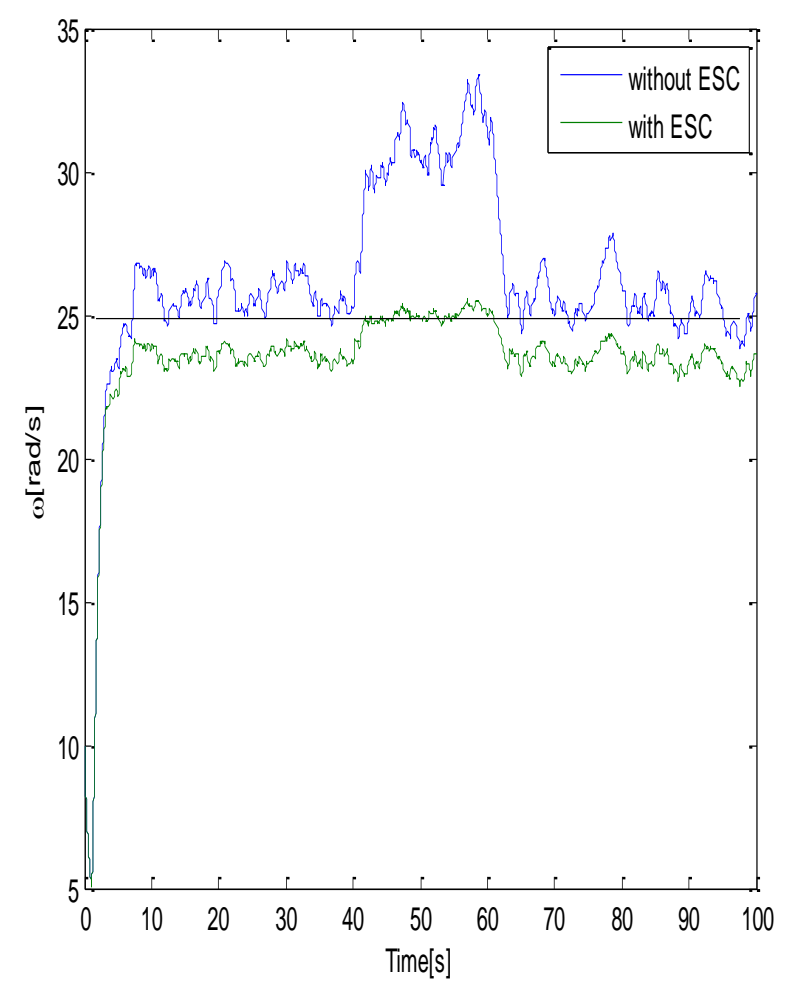

Fig.7. Angular velocity with ESC and without ESC

\section{Results}

First of all Fig. 6 shows the input wind to small-scaled wind turbine which is Reyleigh distribution with average velocity of $40 \mathrm{~m} / \mathrm{s}$. However, in order to confirm the feasibility and reliability of proposed ESC system, we have increased the wind's velocity average to $60[\mathrm{~m} / \mathrm{s}]$ during the $40[\mathrm{~s}]$ to $70[\mathrm{~s}]$ duration. By this sudden and extreme change we aim to see whether the proposed method works or not. As a consequence, as it is clear in Fig. 7. Angular velocity without ESC system exceeds the boundary angular velocity and when ESC system is loaded angular velocity almost oscillating below the reference angular velocity which is $25[\mathrm{rad} / \mathrm{s}]$. Thus, we could confirm the feasibility and reliability of proposed ESC system that even in extreme high speed wind it can be operated and maintain the stability and enhance the performances.

\section{Conclusion}

In this research, in order to operate small-scaled wind turbine system in the extreme high wind velocity, electromagnetic stall control system has been offered to be implemented and combined with pitch angle control. Unlike the adaptive friction control, electromagnetic stall control system has no thermo energy lost since it has no mechanical connection to the shaft. Moreover, the maintenance is much easier than adaptive friction control system. Thus, we can conclude that it is more economy compare to adaptive friction control system. The most significant matter is that we could confirm that electromagnetic stall control system is robust against extreme strong wind. Thus, small scaled wind turbine system can be operated even in storm day. In order to realize ESC system, we are going to consider the uncertainty of small-scaled wind turbine since in actual case they might be parameters perturbation.

\section{Acknowledgements}

At the top of appreciation list of would like sincerely express my appreciation and gratitude to laboratory members that had been cooperating with this research.

\section{References}

[1] R.C. Dorf, R. H .Bishop "Modern Control System” Prentice Hall, 2002

[2] G.J Balas, J.C Doyle, K Glover, A. Packard, R Smith "Robust Control Toolbox TM 3 User's Guide" The Math Works

[3] Sigurd Skogestad, Ian Postlethwaite "Multi Variable Feedback Control Analysis and Design" JOHN WIEY \& SONS, 1996

[4] Miroslav Krstic, Ioannis Kanellakopoulos, Petar Kokotovic, "Nonlinear and Adaptive Control Design" John WILEY \& SONS, INC. 1995

[5] Alberto Isidori, "Nonlinear Control System", Springer 1995 